\title{
In-vitro biocompatibility testing of phycobiliproteins from Gracilaria changii
}

\author{
Rugi Vicente Rubi ${ }^{1, *}$, Erison Roque ${ }^{1}$, Fritzzie Gutierrez ${ }^{1}$, May Anne Hegina ${ }^{1}$, Jeanell Martin ${ }^{1}$, Arrianne Jaye Mata ${ }^{1}$, and \\ Ervin Mission ${ }^{1}$
}

${ }^{1}$ Department of Chemical Engineering, Adamson University, Manila, Philippines

\begin{abstract}
The present study probes into the biocompatibility testing of Phycobiliproteins (PBPs) from Gracilaria changii, in-vitro. The cell survivability and cytotoxicity of PBPs to cultured Human Cervix Carcinoma (Hep-2C) cells were tested and observed using MTT (3-(4, 5- Dimethylthiazol-2-yl)-2, 5diphenyltetrazolium bromide) assay. For ten-fold dilutions, Phycobiliproteins (PBPs) promotes highest cell survivability at $10-4$ dilution rate with $239.62 \%$ and highest cytotoxic effect, with $40.21 \%$, at $10-1$ dilution. The compositions of phycobiliproteins were determined using Ultraviolet (UV-Vis) spectrophotometer giving visible absorbance peaks at 498.5, 614 and $651 \mathrm{~nm}$ for the three PBPs composition: Phycoerythrin (PE), Phycocyanin (PC) and Allophycocyanin (APC), respectively. The effects of time duration in centrifugation process of Phycobiliproteins (PBPs) from Gracilaria changii in 3, 5 and 7 minutes, were proven significant on the quantity of the extracted PBPs. The highest average concentration of $20.37 \mu \mathrm{g} / \mathrm{ml}$ for APC, $13.31 \mu \mathrm{g} / \mathrm{ml}$ for PC and $18.57 \mu \mathrm{g} / \mathrm{ml}$ for PE were obtained at the 5 minute-extraction process.
\end{abstract}

\section{Introduction}

The rising demand for natural instead of synthetic materials especially in biomedical applications where high biocompatibility and no adverse effects for the host organism are required (Mano et al. 2007), has led to an outburst of scientific papers involved in the study of biobased materials. In this view, the feasibility of using starting materials obtainable from organic sources has seen potential for such purpose. A great potential for reliable and sustainable source for the production of biochemically active compounds such as phycobiliproteins are macroalgae.

Phycobiliproteins (PBPs), consisting of phycoerythrin (PE), phycocyanin (PC) and allophycocyanin (APC), are the principal photoreceptors for photosynthesis in bluegreen and red as well as some other types of macro algae. These proteins with covalently linked bile pigment chromophores may comprise more than $60 \%$ of the total soluble cellular protein or almost $20 \%$ of the total dry weight of one alga (Bogorad 1975). Currently, PBPs are mainly used as fluorescent probes that are expensive after commercialization (Cai et al. 2012); Furthermore, the effects of PBPs on anti-oxidation, anti- tumor, anti-virus, anti-inflammation, liver-protection, neuro-protection, UV protection, atherosclerosis and skin function activating have been demonstrated.

PBPs were used as photo synthesizers in different other biomedical applications. These require tests that involve the exposure of substances extracted from test material to one of two cell culture lines. Cell cultures are extremely sensitive to minute quantities of leachable chemicals and readily display characteristic signs of toxicity in the presence of potentially harmful leachable. Cytotoxicity in vitro testing is also required in testing the biocompatibility of materials. The protein assay is an indirect measurement of cell viability since it measures the protein content of viable cells that are left after washing of the treated plates. Phycobiliproteins from different macro algae had been an interesting topic for major breakthroughs in biomedical research. It was found out that diverse groups of red seaweeds had significant amount of Phycobiliproteins in it. Based on researches, they are used as photosensitizers for treatment of tumors and have potential to substitute Photofrin (a kind of light sensitive agent in photodynamic therapy) in common use which is purified from animal blood (Francavilla et al. 2013). Phycobiliproteins are a group of colored proteins present commonly on red seaweeds. They are extensively commercially used in foods, cosmetics, biotechnology, pharmacology, and medicine. They also have high potential pharmaceutical and biological properties (Manirafasha et al. 2016).

In this study, we used the extracted and purified amount of Phycobiliproteins from Gracilaria changii which is abundant in the Philippines. Phycobiliproteins from Gracilaria changii, can be expensive, for about 50 euros (P 2,850.00) per milligram. Since the cultivation of the Gracilaria changii is abundant in the southernmost part of Luzon Island in the Philippines, it will be a good start for conducting researches concerning

\footnotetext{
*Corresponding author: erison.roque@adamson.edu.ph
} 
Phycobiliproteins from the said seaweed. In line with this, Phycobiliproteins from Gracilaria changii (Red seaweed), will be considered as a natural source of biomaterial that will be tested for its in- vitro biocompatibility for potential biomedical applications by assessing its cytotoxicity and cell survivability using MTT assay.

\section{Materials and methods}

\subsection{Cells and materials}

In the preparation and lyophilization of the red seaweed Gracilaria changii, a Labconco Freeze Dry System Freezone 4.5 was used to freeze-dry the seaweeds. For the extraction of the phycobiliproteins, freeze dried algal sample mixed with alumina was ground manually using a pestle and mortar. Incubation with buffer of the sample was done using a homogenizer. A micro centrifuge glass tube was used to contain the mixture and Hanil Miro-I2 centrifuge machine operating at 13,500 rpm was used for centrifugation. Phycobiliproteins identified as phycoerythrin (PE), phycocyanin (PC) and allophycocyanin (APC) were quantified in concentration $(\mu \mathrm{g} / \mathrm{mL})$ using PerkinElmer Lambda 25 Ultraviolet (UVVis) spectrophotometer. In the purification of phycobiliproteins, an ammonium sulfate nomogram was used to determine the amount of $\left(\mathrm{NH}_{4}\right)_{2} \mathrm{SO}_{4}$ which will yield the desired $\%$ saturation. The collected supernatant with $\left(\mathrm{NH}_{4}\right)_{2} \mathrm{SO}_{4}$ was mixed using a magnetic stirrer. In the dialysis of phycobiliproteins, dialysis membrane (Spectrum Labs), glass stirring rods and 2L Pyrex beakers were used. In the MTT assay, the mixture was transferred in microplate wells containing $70-80 \%$ confluent Human Cervix Carcinoma (Hep-2C) cells and optical density was read using Bio-Rad iMark microplate reader. The percent cell cytotoxic effect (\%CTE) and percent cell survival (\%CS) was computed. Non-linear fit of concentration versus response was created using GraphPad Prism 7.

\subsection{Chemicals and standard solutions}

Phycobiliproteins were extracted from the freeze-dried algal sample using $1 \mathrm{M}$ acetic acid-sodium acetate buffer (pH 5.5) with $0.01 \%$ v/v of sodium azide. The mixture was purified and brought to the desired saturation with ammonium sulfate $\left(\mathrm{NH}_{4}\right)_{2} \mathrm{SO}_{4}$. The resulting precipitate from the sample was mixed and dissolved in $1 \mathrm{mM}$ of $10 \mathrm{~mL}$ sodium phosphate $(\mathrm{NaPi})$ Buffer with $1 \mathrm{M}$ sodium chloride $(\mathrm{NaCl})$ and was dialyzed against $1 \mathrm{mM} \mathrm{NaPi}$ buffer that contained $0.1 \mathrm{M} \mathrm{NaCl}$ at $\mathrm{pH}$ 7.0. In the MTT assay, an 8- series of 10-fold dilution was prepared using the carrier phosphate buffered saline (PBS). Minimum Essential Medium with 2\% Fetal Bovine Serum was used as neutralizer. After incubation, plates were washed with PBS thrice before the addition of the MTT solution $(5 \mathrm{mg} / \mathrm{ml})$. Plates were incubated before the addition of dimethyl sulfoxide.

\subsection{Biomass sampling}

About $3 \mathrm{~kg}$ of wet biomass were sampled respectively, algal biomass was washed with distilled water and their epiphytes removed. The cleaned seaweed was freeze-dried using Labconco Freeze Dry System/ Freezone 4.5 at $-110^{\circ} \mathrm{C}$ for 5 days and then ground to fine powder and stored in airtight containers at $20^{\circ} \mathrm{C}$.

\subsection{Phycobiliprotein extraction and analysis}

An amount of fine freeze-dried sample mixed with alumina was prepared. The mixture was suspended in $1 \mathrm{M}$ acetic acid-sodium acetate buffer $(\mathrm{pH} 5.5)$ with $0.01 \% \mathrm{v} / \mathrm{v}$ of sodium azide for $20 \mathrm{~min}$ in the dark. After the incubation with buffer, samples were pounded manually using mortar and pestle. The mixture was transferred to a micro centrifuge glass-tube and was centrifuged using Hanil Miro-I2 centrifuge machine operating at 13,500 rpm per $g$ for 3, 5 and 7 mins. Supernatant was collected and the pellet was extracted three times again with buffer as described. Supernatants were combined and the final volume of the extract was about $80.0 \mathrm{~mL}$.

Phycobiliproteins (identified as R-phycoerythrin (PE), phycocyanin (PC) and allophycocyanin (APC)) were quantified in concentration $(\mu \mathrm{g} / \mathrm{mL})$ using PerkinElmer Lambda 25 Ultraviolet (UV-Vis) spectrophotometer. Based on the journal (Kursar et al. 1983) since visible absorbance of the aqueous supernatants was determined at the same range of wavelength as (Kursar et al. 1983) which is the 498.5, 614.0, and $651.0 \mathrm{~nm}$. Their APC, PC, and $\mathrm{PE}$ contents were calculated as, ug/ml using the following equations:

$$
\begin{gathered}
\mathrm{APC}=181.3 \mathrm{~A}_{651}-22.3 \\
\mathrm{PC}=151.1 \mathrm{~A}_{614}-99.1 \mathrm{~A}_{651} \\
\mathrm{PE}=155.8 \mathrm{~A}_{498.5}-40 \mathrm{~A}_{614}-10.5 \mathrm{~A}_{651}
\end{gathered}
$$

\subsection{Ammonium sulfate precipitation}

The collected supernatant with the addition of $\left(\mathrm{NH}_{4}\right)_{2} \mathrm{SO}_{4}$ was mixed using magnetic stirrer for 40 minutes to achieve $25 \%$ saturation. The mixed sample with $\left(\mathrm{NH}_{4}\right)_{2} \mathrm{SO}_{4}$ was transferred in micro centrifuge tubes and centrifuged for 15 minutes. The volume of the supernatant was determined and the pellets were discarded. The collected supernatant was then added again with $\left(\mathrm{NH}_{4}\right)_{2} \mathrm{SO}_{4}$ and mixed for 40 minutes to achieve $50 \%$ saturation. Sample was again centrifuged for 15 minutes and the supernatant was removed using a dropper and the pellets were collected.

\subsection{Dialysis of phycobiliproteins}

The precipitate so obtained at each step of precipitations was dialyzed overnight against Sodium phosphate $(\mathrm{NaPi})$ buffer with Sodium chloride $(\mathrm{NaCl})$ at $4^{\circ} \mathrm{C}$. The dialysis membrane (Spectrum Labs) was first prepared by submerging in deionized water solution for 20 minutes. One millimolar of $\mathrm{NaPi}$ Buffer with $1 \mathrm{M} \mathrm{NaCl}$ (sodium chloride) was mixed to the pellets. The sample was loaded 
into the dialysis tubing. After that, the dialysis tubing containing the sample was submerged to a beaker containing $1 \mathrm{mM} \mathrm{NaPi}$ buffer that contained $0.1 \mathrm{M} \mathrm{NaCl}$ at $\mathrm{pH}$ 7.0. It is dialyzed for at room temperature. Then the buffer was changed and dialyzed again for 2 hours. The last buffer change was dialyzed overnight at $4^{\circ} \mathrm{C}$. Appendix E shows the phycobiliproteins after dialysis.

\subsection{Storage of phycobiliproteins}

Dialyzed phycobiliproteins was stored in $5 \% \mathrm{NaCl}$ (sodium chloride) for testing at $4{ }^{\circ} \mathrm{C}$ under dark condition.

\subsection{Statistical analysis}

All experiments were performed in triplicate. Design of experiment was generated and analyzed by the software Minitab17. Statistical differences between experimental results were assessed by one- factor ANOVA together with the statistical evaluation for the one factor of this study (time) at each three trials.

\subsection{Cytotoxicity test: Measure cell viability of cultured cells}

Human cervix carcinoma (Hep-2C) cells previously washed three times with phosphate buffer solution were seeded into wells of a 96-well culture plate and incubated in $37^{\circ} \mathrm{C}$ in $5 \% \mathrm{CO} 2$ and $98 \%$ humidity for 24 hours. After cell attachment, $100 \mu \mathrm{l}$ volume of this mixture was transferred in microplate wells containing 70-80\% confluent Hep-2C cells. Tenfold serial dilution was prepared using the carrier as cytotoxic control. Each dilution was tested in triplicates. After 72 hours of incubation, plates were washed with phosphate buffer solution thrice before the addition of $15 \mu$ l MTT solution $(5 \mathrm{mg} / \mathrm{ml})$. Plates were incubated for another 3 hours before the addition of dimethyl sulfoxide. Plates were incubated for another 15 minutes and then optical density was read at $595 \mathrm{~nm}$ Bio-Rad iMark microplate reader.

\section{Results and discussion}

\subsection{Absorption phycobiliproteins spectrophotometer}

\section{spectra of using}

Fig. 1(a) shows the absorption spectra of phycobiliprotein gained after three trials of extraction with 3-minute centrifugation time duration; Fig. 1(b) shows the absorption spectra of phycobiliprotein gained after three trials of extraction with 5-minute centrifugation time duration; and Fig. 1(c) shows the absorption spectra of phycobiliprotein gained after three trials of extraction with 7-minute centrifugation time duration. The PE of the aqueous extract of $G$. changii the APC of the aqueous extract of $G$. changii has absorption peaks in the visible region at $651 \mathrm{~nm}$.

Table 1 shows the average values of the absorption peaks of phycobiliprotein gained using UV- Vis spectrophotometer after performing three trials of extraction with 3, 5 and 7 minutes of centrifugation. Due to high absorption coefficients and quantum yields of phycobiliproteins, absorption peaks obtained using UVVis spectrophotometer became visible at the reference wavelength of Phycoerythrin $(\lambda \max =498.5 \mathrm{~nm})$, Phycocyanin $(\lambda \max =614 \mathrm{~nm})$ and Allophycocyanin $(\lambda \max =651 \mathrm{~nm})$. Furthermore, it was proven that there is presence of the three Phycobiliprotein classes, PE, PC and $\mathrm{APC}$ in the extraction part.

(a)
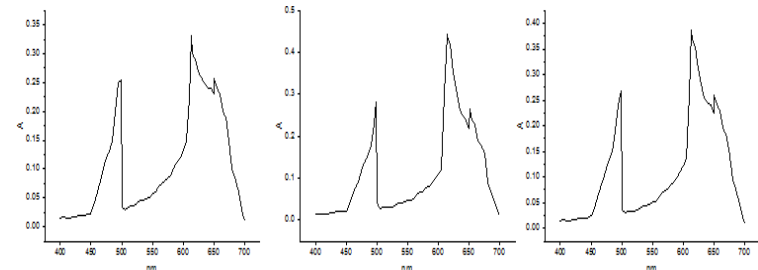

(b)
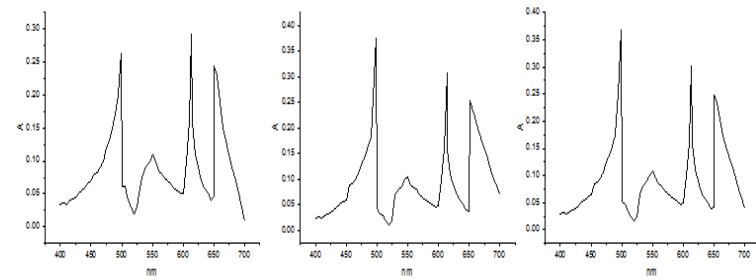

(c)
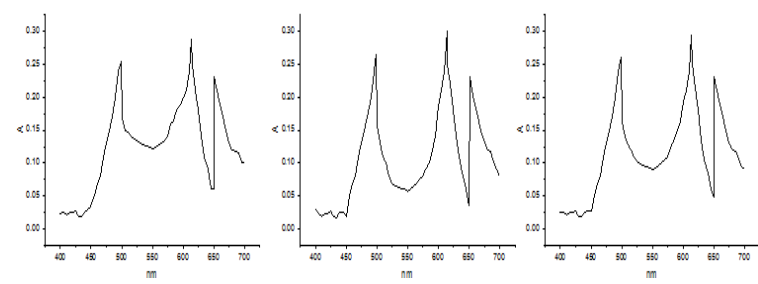

Fig. 1. Absorption spectra of extracted phycobiliproteins allophycocyanin (apc), phycocyanin (pc) and r-phycoerythrin (pe) from G. changii at (a) 3 minutes (b) 5 minutes (c) 7 minutes of centrifugation

Table 1. Average values of absorption peaks of extracted phycobiliproteins at 3,5 and 7 minutes of centrifugation.

\begin{tabular}{|c|c|c|c|}
\hline Wavelength $(\mathrm{nm})$ & $3 \mathrm{~min}$ & $5 \mathrm{~min}$ & $7 \mathrm{~min}$ \\
\hline 498.5 & 0.064 & 0.178 & 0.152 \\
\hline 614 & 0.089 & 0.163 & 0.163 \\
\hline 651 & 0.053 & 0.134 & 0.127 \\
\hline
\end{tabular}

\subsection{Absorption spectra of purified phycobiliproteins spectrophotometer \\ UV-Vis}

Fig. 2(a) shows the absorption spectra of phycobiliprotein gained after three trials of purification with 3-minute centrifugation time duration; Fig. 2(b) shows the absorption spectra of phycobiliprotein gained after three trials of purification with 5-minute centrifugation time duration; and Fig. 2(c) shows the absorption spectra of phycobiliprotein gained after three trials of purification with 7-minute centrifugation time duration. The PE of the purified aqueous extract of $G$. changii has absorption peaks in the visible region at $498.5 \mathrm{~nm}$; the PC of the purified aqueous extract of $G$. changii has absorption peaks in the visible region at $614 \mathrm{~nm}$; and the APC of the 
purified aqueous extract of $G$. changii has absorption peaks in the visible region at $651 \mathrm{~nm}$. (a)

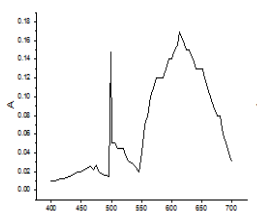

(b)

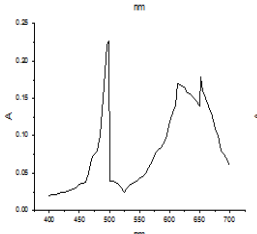

(c)

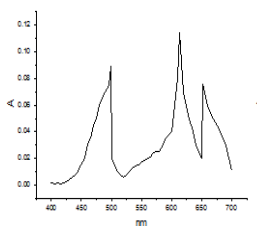

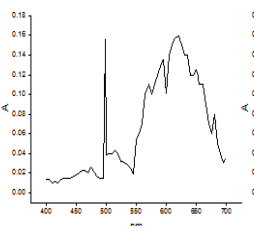
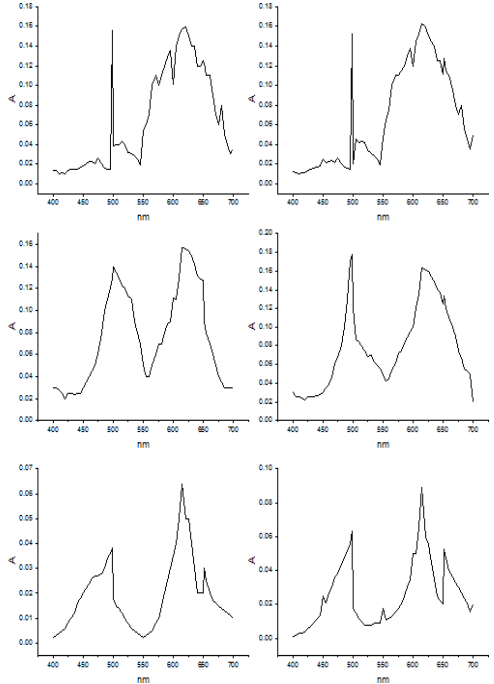

Fig. 2. Absorption Spectra of purified phycobiliproteins Allophycocyanin (APC), phycocyanin (PC) and R-phycoerythrin (PE) from G. changii at (a) 3 (b) 5 and (c) 7 minutes of centrifugation

Table 2. Average values of absorption peaks of extracted phycobiliproteins at 3,5 and 7 minutes of centrifugation

\begin{tabular}{|c|c|c|c|}
\hline Wavelength $(\mathrm{nm})$ & $3 \mathrm{~min}$ & $5 \mathrm{~min}$ & $7 \mathrm{~min}$ \\
\hline 498.5 & $\underline{0.268}$ & $\underline{0.269}$ & $\underline{0.260}$ \\
\hline 614 & $\underline{0.387}$ & $\underline{0.301}$ & $\underline{0.294}$ \\
\hline 651 & $\underline{0.261}$ & $\underline{0.249}$ & $\underline{0.231}$ \\
\hline
\end{tabular}

Table 2 shows the average values of the absorption peaks of phycobiliprotein gained using UV- Vis spectrophotometer after performing three trials of purification with 3,5 and 7 minutes of centrifugation. Due to high absorption coefficients and quantum yields of phycobiliproteins, absorption peaks obtained using UVVis spectrophotometer became visible at the reference wavelength of Phycoerythrin $(\lambda \max =498.5 \mathrm{~nm})$, Phycocyanin $(\lambda \max =614 \mathrm{~nm})$ and Allophycocyanin $(\lambda \max =651 \mathrm{~nm})$. Furthermore, it was proven that there is presence of the three Phycobiliprotein classes, PE, PC and APC in the extraction part.

\subsection{Effect of time durations in phycobiliprotein concentration using Minitab17}

Table 3 and 4 presented a one-way ANOVA of the effect of time durations 3,5 and 7 minutes on the average concentration of extracted and purified phycobiliproteins. The significance of time duration in the extraction process were compared to the significance of time duration in the purification process. There was a significant effect of time on extracted phycobiliprotein concentration at the $p<0.05$ level $[F(2)=9.52, p=0.014]$ while an insignificant effect on purified phycobiliprotein concentration at the $p>0.05$ level $[F(2)=0.75, p=$ 0.513]. Taken together, these results suggest that time duration influence phycobiliprotein concentration and none on the purified part. Hence, we can disregard the effect of time in the purification part.

Table 3. ANOVA result of the effect of time duration 3, 5 and 7 minutes on average extracted phycobiliprotein concentration.

\begin{tabular}{cccccc}
\hline Source & $\begin{array}{c}\text { Degrees } \\
\text { of } \\
\text { freedom }\end{array}$ & $\begin{array}{c}\text { Adjusted } \\
\text { sum of } \\
\text { squares }\end{array}$ & $\begin{array}{c}\text { Adjusted } \\
\text { mean } \\
\text { square }\end{array}$ & F-Value & P-Value \\
\hline TIME & 2 & 180.71 & 90.353 & 9.52 & 0.0 \\
Total & 8 & 237.67 & & & 14 \\
\hline
\end{tabular}

Table 4. ANOVA result of the effect of time duration 3, 5 and 7 minutes on average purified phycobiliprotein concentration.

\begin{tabular}{cccccc}
\hline Source & $\begin{array}{c}\text { Degrees } \\
\text { of } \\
\text { freedom }\end{array}$ & $\begin{array}{c}\text { Adjusted } \\
\text { sum of } \\
\text { squares }\end{array}$ & $\begin{array}{c}\text { Adjusted } \\
\text { mean } \\
\text { square }\end{array}$ & F-Value & P-Vahue \\
\hline TIME & 2 & 67.56 & 33.78 & 0.75 & 0.513 \\
Total & 8 & 38.73 & & & \\
\hline
\end{tabular}

\subsection{Effect of centrifugation time durations in extracted phycobiliprotein concentration}

Fig. 3 shows the average concentration of the extracted phycobiliproteins affected by 3,5 and 7-minute time duration, which revealed that 5 minutes was the optimal time obtaining the highest concentrations of 20.37, 13.31 and $18.57 \mu \mathrm{g} / \mathrm{mL}$ for APC, PC and PE, respectively. The phycobiliproteins was greatly affected as time was increased to 7 minutes as the residual concentrations for phycobiliproteins was decreased to 7.62, 8.20 and $5.55 \mu \mathrm{g} / \mathrm{mL}$ for APC, PC and PE, respectively. These results work under the theory of the disruption method used for the cell wall and cell membrane which effectively releases the phycobiliproteins into the extracting medium. However, it can also destroy the phycobiliproteins if longer disruption time were used due to higher strain and heat accumulation. Similarly, the work of (Munier et al. 2015 ) is associated with the need for standardization of time recovery of phycobiliproteins in biomass to evaluate metabolite accumulation as it was considered for determining the best time period for cell growth. Thus, the comparative analyses between the phycobiliproteins at different time durations $(3,5$ and 7 minutes) were performed on the extraction method using the red macroalgae G. changii.

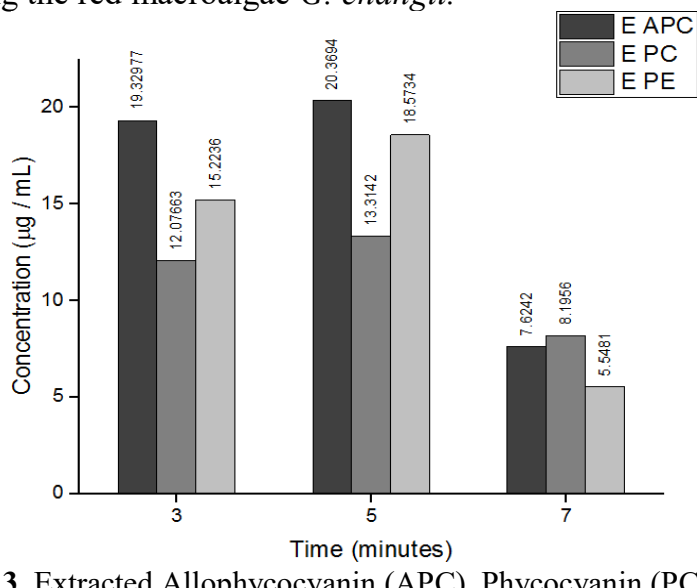

Fig. 3. Extracted Allophycocyanin (APC), Phycocyanin (PC) and Phycoerythrin (PE) with duration of time 3, 5 and 7 minutes. (a) Average values of Phycobiliproteins concentration 
Table 5. Effect of Phycobiliprotein 1 on Hep-2C Cell Survival and Cytotoxicity

\begin{tabular}{ccc}
\hline Dilution & \%CS & \%CTE \\
\hline $10^{0}$ & 64.25 & 35.75 \\
\hline $10^{-1}$ & 86.74 & 13.26 \\
\hline $10^{-2}$ & 73.39 & 26.61 \\
\hline $10^{-3}$ & 216.97 & -116.97 \\
\hline $10^{-4}$ & 198.36 & -98.36 \\
\hline $10^{-5}$ & 226.96 & -126.96 \\
\hline $10^{-6}$ & 119.64 & -19.64 \\
\hline $10^{-7}$ & 123.77 & -23.77 \\
\hline $10^{-8}$ & 186.56 & -86.56 \\
\hline
\end{tabular}

Cytotoxic effect of Phycobiliprotein 1 on Hep2-C

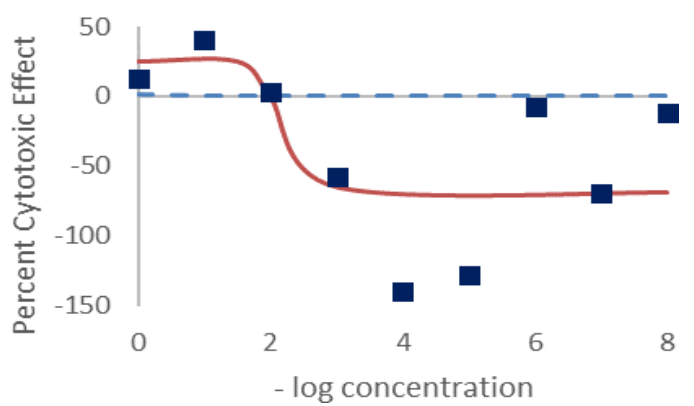

Fig. 4. Cytotoxic Effect of Phycobiliprotein 1 on Hep$2 \mathrm{C}$.

The cell survival of Hep-2C cells and cytotoxic effect of Phycobiliprotein 1 from Gracilaria changii against Hep-2C cells shows a dose dependent inhibitory effect, as shown in Fig. 4.

The decrease of phycobiliprotein concentration as shown in Table 5 presented that the survival rates of Hep$2 \mathrm{C}$ were at most at 10-4 with a value of $239.62 \%$ and least at $10-1$ with a value of $59.79 \%$, respectively, showing a dose-dependent effect. Phycobiliprotein 1 was most cytotoxic towards Hep-2C cells when diluted to 10-1-fold and least cytotoxic at 10-4-fold dilution. The percent survival of Hep-2C cells which reduced to $87.44 \%$ and $59.79 \%$, increased to $96.97 \%, 157.95 \%$ and $239.62 \%$, reduced to $227.57 \%$ and $107.32 \%$, then reduced again to $168.96 \%$ and $111.83 \%$, respectively, for 10 -fold dilution, correspond to the baseline survival rate of the nontreatment group. In simple words, at the first two dilutions, it has inhibitory effect on the Hep-2C cells, while at the next three dilutions; the phycobiliproteins encouraged growth in the Hep-2C cells. Moreover, it shows that the cytotoxicity of phycobiliproteins at 10 -fold dilutions has opposite result on the Hep-2C cells.

The results showed that there is a certain dilution rate that affects the behavior of Phycobiliprotein 1 concentration as was also proven by(Wang et al. 2012), in which they expressed that the cell demise reaction to Photodynamic treatment (PDT) of phycobiliprotein differs because of the physical properties of the photosensitizer utilized, the PDT measurements and the cell sort and inhibits the growth of Hep-2C cells, as was proven by
Zhang et al. (2011), in which they stated that the photosensitizers can absorb luminous energy and convert oxygen to singlet oxygen $(1 \mathrm{O} 2)$, a powerful reactive oxygen species (ROS) that can kill tumor cells. It was observed that the concentration of the liquid sample phycobiliprotein 1 and the host cell line Hep-2C which was used in the testing are the factors that can affect the behavior of the sample to the cells.

Table 6. Effect of Phycobiliprotein 2 on Hep-2C Cell Survival and Cytotoxicity

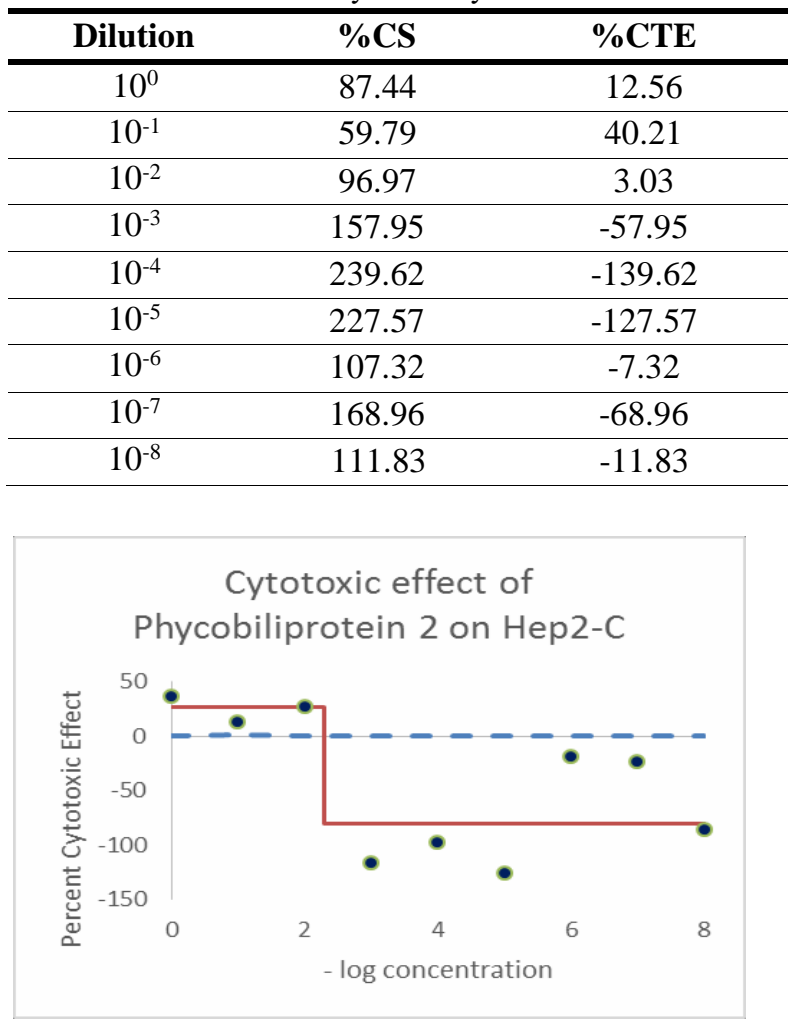

Fig. 5. Cytotoxic Effect of Phycobiliprotein 2 on Hep-2C.

The cell survival of Hep-2C cells and cytotoxic effect of Phycobiliprotein 2 from Gracilaria changii against Hep-2C cells shows a dose dependent inhibitory effect, as shown in Fig. 5. Phycobiliprotein concentration as shown in Table 3.4 presented that the decrease of phycobiliprotein concentration, the survival rates of Hep$2 \mathrm{C}$ were at most at $10-5$ with a value of $226.96 \%$ and least at 100 with a value of $64.25 \%$, respectively, showing a dose-dependent effect. Phycobiliprotein 2 was most cytotoxic towards Hep-2C cells when undiluted and least cytotoxic at 10-5-fold dilution. The percent survival of Hep-2C cells which decreased from $86.74 \%$ to $73.39 \%$ under 10-1 and 10-2 dilutions and increased from $198.36 \%$ to at its highest peak with a value of $226.96 \%$ under $10-4$ and 10-5 dilutions of phycobiliproteins, respectively, correspond to the baseline survival rate of the nontreatment group. In simple words, at the first three dilutions, Phycobiliprotein has inhibitory effect on the Hep-2C cells, while at the next three dilutions; the phycobiliproteins encouraged growth in the Hep-2C cells. Moreover, it shows that the cytotoxicity of phycobiliproteins at 10 -fold dilutions has opposite result on the Hep-2C cells. 
The results show that there is a certain dilution rate that affects the behavior of Phycobiliprotein 2 concentration as was also proven by (Wang et al. 2012), in which they expressed that the cell demise reaction to Photodynamic treatment (PDT) of phycobiliprotein differs because of the physical properties of the photosensitizer utilized, the PDT measurements and the cell sort and inhibits the growth of Hep-2C cells, as was proven by (Zhang et al. 2011) in which they stated that the photosensitizers can absorb luminous energy and convert oxygen to singlet oxygen (1O2), a powerful reactive oxygen species (ROS) that can kill tumor cells. It was observed that the concentration of the liquid sample phycobiliprotein 2 and the host cell line Hep-2C which was used in the testing are the factors that can affect the behavior of the sample to the cells.

\section{Conclusion}

\subsection{Absorption spectra of extracted and purified phycobiliproteins using UV-Vis spectrophotometer}

Absorption peaks obtained using UV- Vis spectrophotometer is visible at the reference wavelength of Phycoerythrin $(\lambda \max =498.5 \mathrm{~nm})$, Phycocyanin $(\lambda \max =$ $614 \mathrm{~nm})$ and Allophycocyanin $(\lambda \max =651 \mathrm{~nm})$. Thus, it is proved that there is presence of the three Phycobiliprotein classes; PE, PC and APC in the extraction and purification part. It can be used to prove that there is presence of the three Phycobiliprotein classes, APC, PC and PE in the extraction and purification part. Hence, one of the primary criteria for the presence of phycobiliproteins in the macro algae are the spectroscopic properties of the extracted and purified pigment-proteins.

\subsection{Effect of different centrifugation time duration in concentration of phycobiliproteins}

Effect of centrifugation time duration for triplicate trials in the extraction process of 3,5 and 7 minutes shows significant effect on the average percent yield of phycobiliprotein. Disruption method used for the cell wall and cell membrane effectively releases the phycobiliprotein into the extracting medium that gave a reading of $20.37,13.31$ and $18.57 \mu \mathrm{g} / \mathrm{mL}$ for $\mathrm{APC}, \mathrm{PC}$ and $\mathrm{PE}$, respectively at 5 minutes. Based on the data gathered using the equation of (Kursar et al. 1983), centrifugation at 5 minutes gave the highest yield of phycobiliprotein with $5 \%$ to $30 \%$ higher than at 3 minutes and $50 \%$ to $70 \%$ higher than of 7 minutes. Thus, it was also observed that longer disruption time also destroyed the phycobilins due to higher strain and heat accumulation.

\subsection{Biocompatibility testing of phycobiliprotein using MTT assay for Hep-2C cell}

As MTT assay is used as a method to test cell growth rate and toxicity of the culture cell line. It is used to test the biocompatibility of phycobiliproteins (PBPs) to Hep2C cervix carcinoma. Data show that PBPs promote highest cell survivability at 10-4 dilution with $239.62 \%$ and highest cytotoxic effect, with $40.21 \%$, at 10-1 dilution. Therefore, the higher the concentration PBPs, the more it becomes cytotoxic. The inhibitory effect of phycobiliprotein is desirable in this type cell since cancer cells contain mutagens that make them reproduce faster than healthy cells. But it is also concluded that there are certain factors that can affect its inhibitory effect. Factors are the concentration of the liquid sample phycobiliprotein 2 , the phosphate buffer solution that was used as a carrier in the MTT assay and the host cell line used in the testing which is the Hep-2C. However, it is detrimental when used with healthy cells. Furthermore, based on the data obtained, we can conclude that Phycobiliprotein is biocompatible to human cells.

We would like to express our deepest gratitude to Adamson University via Center for Research and Development for providing us our needs in making this research possible.

\section{References}

[1] Bogorad, L., 1975. Phycobiliprotein: Complementary Chromatic Adaptation. Ann. Rev. Plant Physiol., 26, pp.369-401.

[2] Cai, C. et al., 2012. Large scale preparation of phycobiliproteins from Porphyra yezoensis using coprecipitation with ammonium sulfate. Natural Science, 4(8), pp.536-543.

[3] Francavilla, M. et al., 2013. The red seaweed gracilaria gracilis as a multi products source. Marine Drugs, 11(10), pp.3754-3776.

[4] Kursar, T. a, van der Meer, J. \& Alberte, R.S., 1983. Light-harvesting system of the red alga Gracilaria tikvahiae. Plant physiology, 73(2), pp.361-369.

[5] Manirafasha, E. et al., 2016. Phycobiliprotein: Potential microalgae derived pharmaceutical and biological reagent. Biochemical Engineering Journal, 109, pp.282-296. Available at: http://dx.doi.org/10.1016/j.bej.2016.01.025.

[6] Mano, J.F. et al., 2007. Natural origin biodegradable systems in tissue engineering and regenerative medicine: present status and some moving trends. Journal of the Royal Society, Interface / the Royal Society, 4(17), pp.999-1030. Available at: http://www.ncbi.nlm.nih.gov/pubmed/17412675\%5C nhttp://www.pubmedcentral.nih.gov/articlerender.fcg i? artid=PMC2396201.

[7] Munier, M. et al., 2015. One-step purification of Rphycoerythrin from the red edible seaweed Grateloupia turuturu. Journal of Chromatography B: Analytical Technologies in the Biomedical and Life Sciences, 992, pp.23-29. Available at:

[8] http://dx.doi.org/10.1016/j.jchromb.2015.04.012.

[9] Wang, C. et al., 2012. ANTI-CANCER EFFECTS OF POLYSACCHARIDE AND PHYCOCYANIN. JOURNAL OF PHOTOCHEMISTRY \& PHOTOBIOLOGY, B: BIOLOGY, 19(4), pp.377- 
382. Available at:

http://dx.doi.org/10.1016/j.jphotobiol.2012.09.001.

[10] Zhang, L. et al., 2011. ANTI-CANCER EFFECTS

OF POLYSACCHARIDE AND PHYCOCYANIN. , 19(4), pp.377-382. 